\title{
APLICACIONES DE LA QUÍMICA SUPRAMOLECULAR: SÍNTESIS DE UN SENSOR CON PROPIEDADES FLUORESCENTES EN SOLUCIÓN Y EN FASE SÓLIDA PARA LA DETECCIÓN SELECTIVA DE LITIO
}

\author{
Applications of supramolecular chemistry: synthesis of fluorescent sensor \\ in solution and in solid phase for selective detection of lithium
}

\section{EPISTEMUS}

ISSN: 2007-8196 (electrónico)

ISSN: 2007-4530 (impresa)

Hisila Santacruz Ortega ${ }^{1}$

Luis Miguel López Martínez 2

Gabriela Ivonne Valenzuela Orduño 3

Rocio Sugich Miranda 4

Enrique F. Velázquez Contreras 5

Recibido: 18 de marzo de 2016,

Aceptado: 30 de mayo de 2016

Autor de Correspondencia:

Dra. Hisila Santacruz Ortega

Correo: hisila@polimeros.uson.mx

\section{Resumen}

El presente trabajo reporta la síntesis de un nuevo sensor fluorescente de cadena abierta tipo biscromofórico con grupos éter y aminos como unidades receptoras y naftaleno como unidad indicadora (L1), y la síntesis del ligante (M1) por el método de síntesis orgánica en fase sólida (SOFS) soportado en resina de Merrifield el cual presenta una estructura similar a L1; para ambos ligantes se llevaron a cabo estudios de complejación por la técnica de fluorescencia con los metales alcalinos $\mathrm{Li}^{+}, \mathrm{Na}^{+}$y K $\mathrm{K}^{+}$. Los estudios de complejación con M1 mostraron un aumento de la fluorescencia en presencia de $\mathrm{Li}^{+}$y Na${ }^{+}$, y una respuesta nula en presencia de $\mathrm{K}^{+}$, mientras que $\mathrm{L} 1$ presenta una respuesta incremento de fluorescencia para $\mathrm{Li}^{+}$, con un aumento de 1.2 veces en la intensidad de la banda emisión de monómero y 6 veces en la banda de emisión de excímero; $\mathrm{Na}^{+}$y $\mathrm{K}^{+}$no afectan la fluorescencia de L1.

Palabras clave: Sensor fluorescente, fluorescencia, monómero, excímero.

\section{Abstract}

This work reports the synthesis of a new fluorescent sensor type open chain bischromophoric, with ether and amino groups as receiver units and naphthalene as indicator unit (L1), and the synthesis of $M 1$ by methodology of synthesis organic phase solid (SOFS) supported in Merrifield resin, which has a similar structure to $L 1$. For both ligands complexation studies by fluorescence technique with alkali metals $\left(\mathrm{Li}^{+}, \mathrm{Na}^{+}\right.$and $\left.\mathrm{K}^{+}\right)$were carried out. Coordination studies with $\mathrm{M} 1$ showed increased fluorescence response in presence of $\mathrm{Li}^{+}$ and $\mathrm{Na}^{+}$and no response in presence of $\mathrm{K}^{+}$, whereas $\mathrm{L} 1$ present an increased fluorescence response for $\mathrm{Li}^{+}$, an increase of 1.2 times in the intensity of monomer emission band and 6 times in the excimer emission band; $\mathrm{Na}^{+}$and $\mathrm{K}^{+}$ did not affect the fluorescence of $L 1$.

Keywords: fluorescent sensor, fluorescence, monomer, excimer. 


\section{INTRODUCCIÓN}

La química supramolecular es un área de investigación de gran interés y es considerada por diversos grupos pieza clave en el área de las ciencias analíticas [1] [2]. Uno de los campos de la química supramolecular se centra en el diseño de ligantes fluorescentes capaces de unirse selectiva y reversiblemente a un analito, lo que resulta en un cambio de las propiedades físicas y químicas del sistema. Las funciones básicas de tales ligantes son los procesos de reconocimiento, auto ensamblaje, catálisis y transporte [1] [3] [4]. Debido a sus propiedades, los ligantes fluorescentes ofrecen importantes y atractivas aplicaciones al campo de la química analítica, de hecho permiten llevar a cabo mediciones en tiempo y espacio real cuando se sintetizan en solución, incluso cuando se utilizan inmovilizados en materiales. Una de las aplicaciones de los sensores basados en fluorescencia es la detección de iones metálicos $\left(\mathrm{Li}^{+}\right.$, $\mathrm{Na}^{+}, \mathrm{K}^{+}, \mathrm{Ca}^{2+}, \mathrm{Mg}^{2+}, \mathrm{Zn}^{2+}, \mathrm{Pb}^{2+}, \mathrm{Al}^{3+}, \mathrm{Cd}^{2+}$, etcétera), sin embargo también pueden ser utilizados para la detección de aniones (haluros, citrato, carboxilatos, fosfatos y ATP) $\mathrm{u}$ otras especies difíciles de reconocer, como especies neutras y gases $\left(\mathrm{O}_{2^{\prime}} \mathrm{CO}_{2^{\prime}} \mathrm{NO}\right.$, etcétera) [5].

Los métodos más comunes para la detección de metales son la espectrometría de absorción atómica, la voltamperometría de redisolución anódica y la espectrometría de masas con fuente de plasma de acoplamiento inductivo. Estos métodos difieren considerablemente en términos de capacidad analítica (límites de detección, precisión), requisitos técnicos (preparación de la muestra, calibración, personal capacitado) y costos, de igual manera se consideran técnicas caras y generalmente sofisticadas que dificultan la medición en tiempo real. En contraste, los métodos basados en fluorescencia ofrecen distintas ventajas en selectividad, sensibilidad, respuesta en tiempo real y bajo costo [6]. En la actualidad se han desarrollado muchos sensores fluorescentes e incluso algunos de ellos son comerciales, sin embargo todavía se necesita diseñar sensores más selectivos y menos perturbables por el medio.

Por otra parte, el litio es un metal alcalino que tiene múltiples aplicaciones, principalmente en la industria del vidrio y la cerámica, en la producción de baterías [7] [8] y con menor frecuencia en la industria farmacéutica en la elaboración de medicamentos para tratar la depresión [9]. La contaminación por baterías y pilas de desecho, así como los daños a la salud que pueden generar el litio contenido en ellas, promueven la necesidad de que estos residuos sean tratados de manera eficaz con la finalidad de recuperar el metal y que pueda ser reutilizado. Actualmente no se conoce ningún estudio que evalúe el impacto al ambiente ocasionado por la utilización y manejo inadecuado de pilas y baterías en México.

Dado que el litio no se produce en México existe además una razón económica para su detección selectiva y recuperación a partir de baterías [7] [8].

Con la finalidad de avanzar en el desarrollo de un método adecuado para la detección y recuperación selectiva de litio, en este trabajo se realizó la síntesis de un nuevo ligante del tipo biscromofórico (L1), el cual posee en su estructura grupos donantes éter y aminos, y presenta como unidad indicadora al naftaleno (Figura 1); también se realizó la inmovilización en fase sólida de un ligante similar a L1 (M1) (Figura 1). Por último, se evaluó la capacidad de L1 y M1 de reconocer los metales alcalinos Li, Na y K por la técnica de fluorescencia.
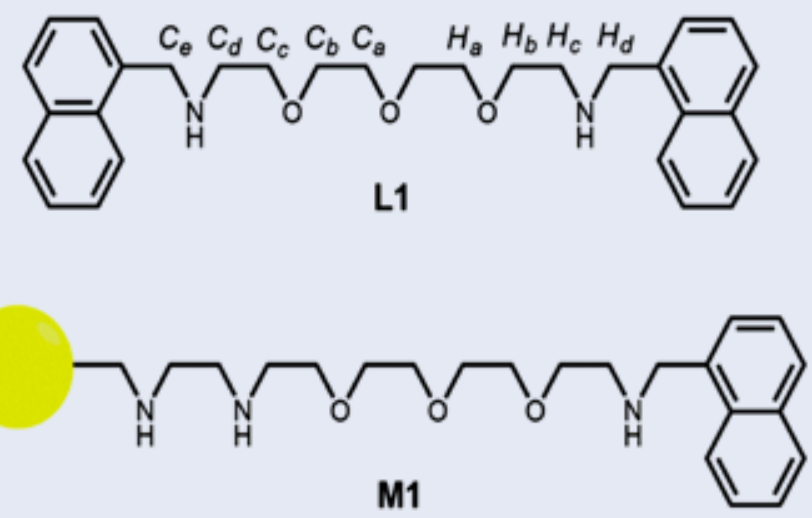

Figura 1. Estructuras de los ligantes sintentizados.

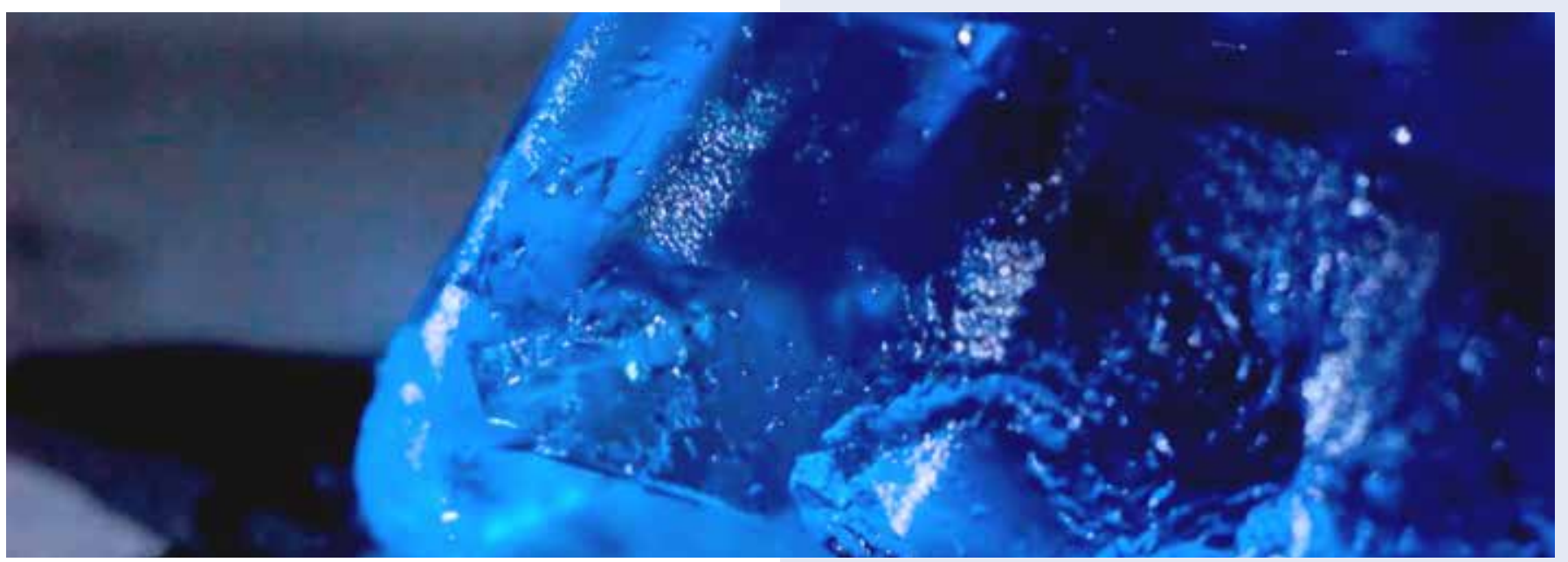




\section{EXPERIMENTAL}

Síntesis del ligante $\mathbf{N}, \mathrm{N}^{\prime}$-((oxibis(etano-2,1-diil))

bis(oxi))bis(metilen))bis(1-(naftalen-1-il)metamina (L1)

La síntesis del ligante L1 se llevó a cabo mediante la reacción entre el bis[2-(2-cloroetoxi)etil]éter con la amina 1-naftalen-il-metilamina. En un matraz de reacción se colocaron $10 \mathrm{~mL}$ de diclorometano (DCM) yse adicionaron el bis[2-(2cloroetoxi)etil]éter $(0.2 \mathrm{mmol})$, la amina 1-naftalenil-metilamina $(0.4 \mathrm{mmol})$ y $\mathrm{K}_{2} \mathrm{CO}_{3}(2.5 \mathrm{mmol})$. La reacción se dejó en agitación constante y a temperatura ambiente por 72 horas (Figura 2). Transcurrido el tiempo de reacción se obtuvo un líquido amarillo el cual fue filtrado para separarlo del $\mathrm{K}_{2} \mathrm{CO}_{3}$, posteriormente el líquido filtrado se evaporó a temperatura ambiente. Una vez seco se obtuvo un líquido viscoso de color café, el cual fue purificado con lavados de etanol/agua en porciones 50/50\%. El producto se secó en estufa de vacío. Una vez obtenido el producto, se caracterizó mediante distintas técnicas espectroscópicas, Infrarrojo (IR), Resonancia magnética nuclear de ${ }^{1} \mathrm{H}$ y ${ }^{13} \mathrm{C}$ (RMN), Ultravioleta visible (UV-Vis), Fluorescencia, además de Espectrometría de masas.
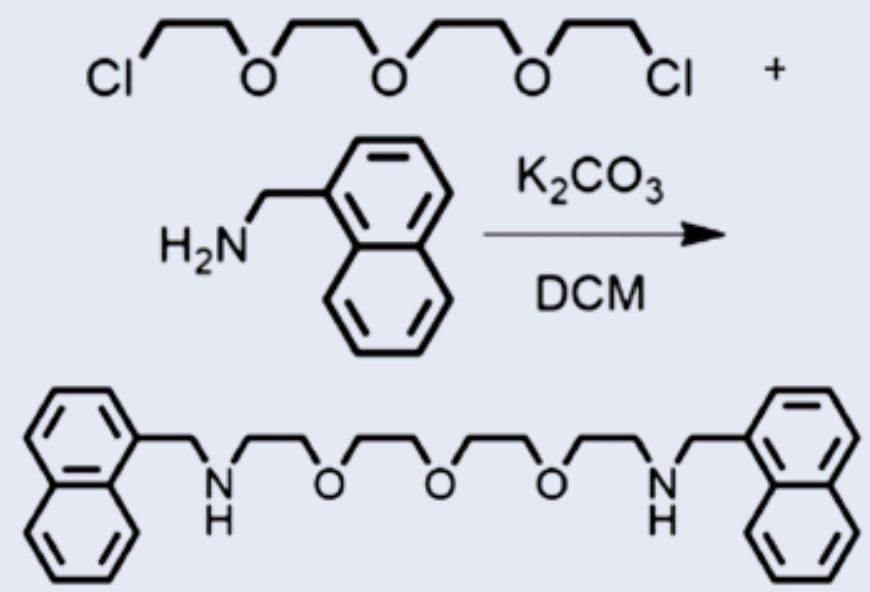

Figura 2. Esquema de la síntesis del ligante L1.

\section{Síntesis del ligante soportado en resina Merrifield (M1)}

La síntesis del ligante $\mathrm{M} 1$ se obtuvo en diferentes pasos mediante la síntesis orgánica en fase sólida (SOFS). Primeramente se lleva a cabo la modificación del espaciador. En un matraz de reacción se colocaron $200 \mathrm{mg}$ de la resina Merrifield y se adicionaron aproximadamente $10 \mathrm{~mL}$ de $\mathrm{N}, \mathrm{N}$-dimetilformamida (DMF), posteriormente se agregó $\mathrm{K}_{2} \mathrm{CO}_{3}(1.5 \mathrm{mmol})$ y etilendiamina $(1.5 \mathrm{mmol})$. El matraz se colocó en una placa de agitación elíptica y se dejó reaccionar por 24 horas. Una vez transcurrido el tiempo de reacción se llevó a cabo la purificación con filtrados y lavados con agua, etanol y acetona. El producto obtenido se secó a vacío por 6 horas. Para determinar que la modificación de la resina se llevó a cabo, se realizó la prueba colorimétrica de Kaiser que sirve para comprobar

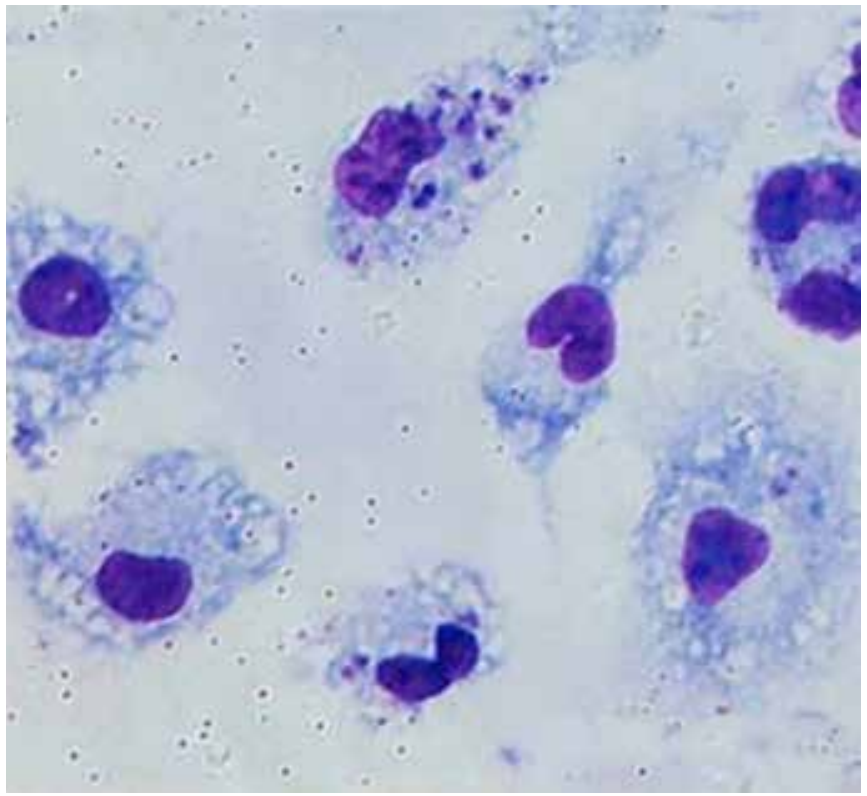

si se encuentran presentes los grupos aminos terminales esperados.

Posteriormente a la modificación del espaciador se inmovilizó la unidad quelante. Se colocaron $150 \mathrm{mg}$ de la resina modificada en un matraz de reacción y se adicionaron $10 \mathrm{~mL}$ de DMF, trietilamina $(1.13 \mathrm{mmol})$ y bis[2(2-cloroetoxi)etil]éter (1.13 mmol). El matraz se colocó en una placa de agitación elíptica por 24 horas, transcurrido el tiempo de reacción se lavó la resina con DCM y éter anhidro. El producto obtenido se secó a vacío por 6 horas.

Por último se llevó a cabo la inmovilización de la unidad indicadora. Se colocó la resina con el grupo quelante (130 $\mathrm{mg}$ ) en un matraz de reacción y se adicionó DMF (10 $\mathrm{mL}), \mathrm{K}_{2} \mathrm{CO}_{3}(1 \mathrm{mmol})$ y 1 -naftalen-metilamina $(1 \mathrm{mmol})$. El matraz se colocó en una placa de agitación elíptica y se dejó reaccionar por 24 horas. Una vez transcurrido el tiempo de reacción el producto se lavó con agua, etanol y acetona, posteriormente se secó a vacío por 6 horas y se caracterizó. La figura 3 muestra el esquema de síntesis del ligante soportado M1. Cada etapa de síntesis se caracterizó por IR y fluorescencia.

\section{Caracterización}

Los espectros de masas se obtuvieron en la Universidad de Arizona, la muestra se analizó por la técnica de ESI, utilizando un equipo de espectrometría de masas de alta resolución marca JEOL modelo XH110A. Los espectros de infrarrojo se obtuvieron en pastillas de $\mathrm{KBr}$, utilizando un espectrómetro FT-IR Perkin-Elmer Modelo Spectrum 2. Los espectros de $\mathrm{RMN}$ de ${ }^{1} \mathrm{H}$ y ${ }^{13} \mathrm{C}$ se obtuvieron a temperatura ambiente en un espectrómetro de resonancia magnética nuclear Bruker Avance 400, utilizando una frecuencia de $400 \mathrm{MHz}$ para ${ }^{1} \mathrm{H}$ y de $100 \mathrm{MHz}$ para ${ }^{13} \mathrm{C}$. Para llevar a cabo la medición por $\mathrm{RMN}$, el compuesto se disolvió en $\mathrm{CDCl}_{3}$. Los espectros de absorción electrónica se obtuvieron en un espectrofotómetro de ultravioleta-visible de 
arreglo de diodos marca Agilent modelo 8545. Todas las mediciones se llevaron a cabo a temperatura ambiente. Los espectros de emisión y de excitación se obtuvieron en un espectrofotómetro de Luminiscencia marca PerkinElmer, modelo LS50B a temperatura ambiente.

\section{Resina Merrifield}
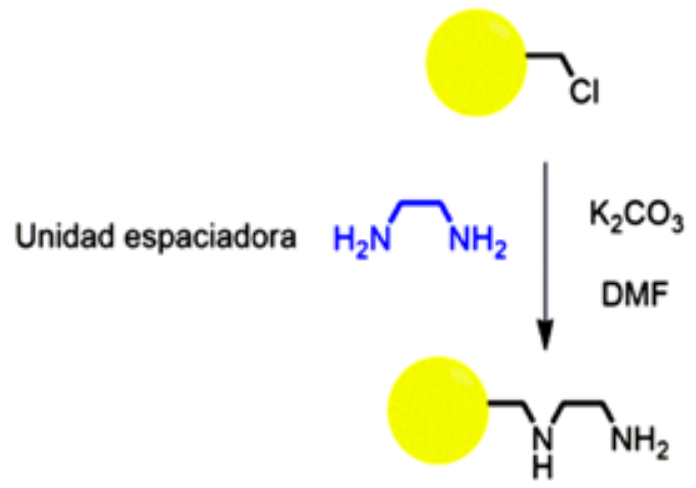

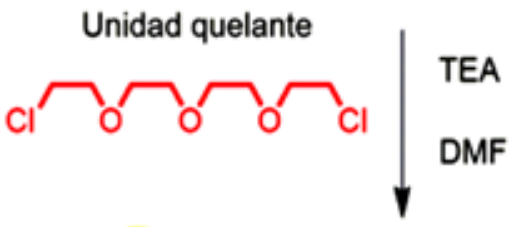<smiles>CCNCCNCCOCCOCCOCCCl</smiles><smiles>NCc1cccc2ccccc12</smiles><smiles>c1ccc(CNCCNCCOCCOCCNCc2cccc3ccccc23)cc1</smiles>

Figura 3. Esquema de la síntesis del ligante soportado M1.

\section{Estudio de coordinación metálica}

\section{del ligante L1 por fluorescencia}

Los estudios de formación de complejos metálicos en solución, se llevaron a cabo por titulaciones espectrofluorimétricas a $25^{\circ} \mathrm{C}$ en soluciones del ligante en buffer MOPS [0.01 M] a pH $=7$. En una celda de cuarzo se colocaron $3.0 \mathrm{~mL}$ de solución del ligante $\mathrm{L} 1\left[1 \times 10^{-6} \mathrm{M}\right]$ y se adicionaron consecutivamente volúmenes de $10 \mu \mathrm{L}$ de solución del cloruro del metal a evaluar, con la finalidad de variar la relación [M]/[L]. Después de cada adición la solución se agitó por 3 minutos utilizando un agitador magnético y enseguida se tomó el espectro emisión a una $\lambda_{\text {ex }}$ de $290 \mathrm{~nm}$ (Figura 4a). Ventana espectral de 300 a 600 $\mathrm{nm}$, utilizando un slit de excitación y de emisión de $5 \mathrm{~nm}$.

\section{Estudio de coordinación metálica}

\section{del ligante soportado M1 por fluorescencia}

La evaluación de la capacidad de aplicación como sensor de M1 se llevó a cabo mediante estudio de fluorescencia utilizando un sistema de flujo (Figura 4b). El cual consistió en colocar de 12-13 mg del material en la celda de flujo y tomar el espectro de fluorescencia, enseguida se le hizo pasar una solución acuosa y se le inyectó $100 \mu \mathrm{L}$ de la solución del metal a evaluar y nuevamente se tomó el espectro de fluorescencia después de cada inyección.
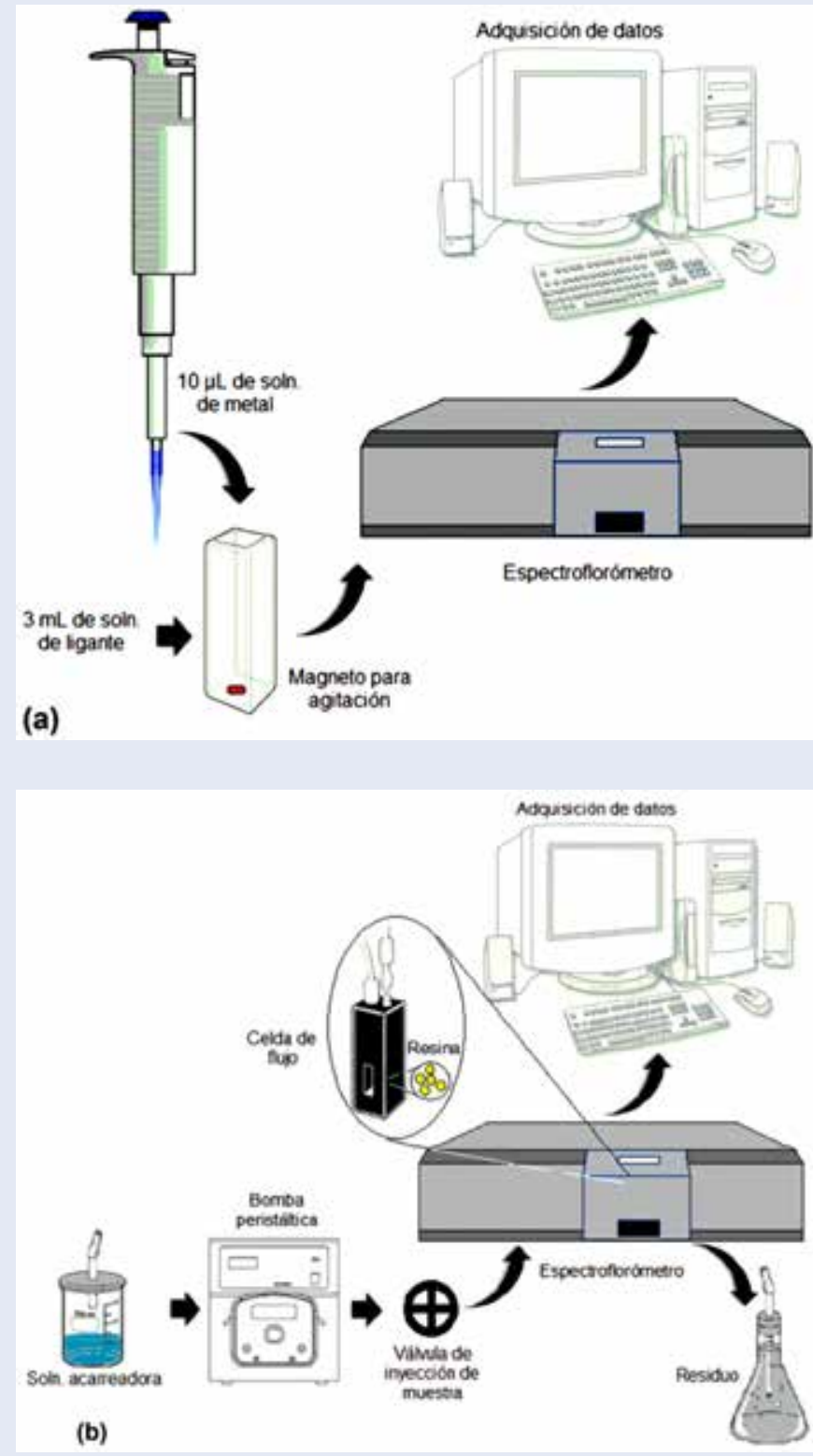

Figura 4. Representación de los estudios de coordinación del ligante L1 (a) y M1 (b) por fluorescencia. 


\section{RESULTADOS}

\section{Síntesis del ligante L1}

De la reacción entre el bis[2-(2cloroetoxi)etil]éter con la amina 1-naftalen-il-metilamina, se obtuvo el ligante de cadena abierta tipo biscromofórico; $\mathrm{N}_{1} \mathrm{~N}^{\prime}$-((oxibis(etano2,1-diil))bis(oxi))bis(metilen))bis(1-(naftalen-1-il)metamina (L1). El nuevo compuesto sintetizado L1 se obtuvo con un rendimiento del $85 \%$ y presentó las siguientes características: se obtuvo como una sustancia viscosa de color café, altamente soluble en acetona y etanol. La caracterización del compuesto a través de las distintas técnicas espectroscópicas confirma la obtención del producto puro. Los espectros de IR muestran bandas vibracionales de estiramientos y flexiones correspondientes a los grupos funcionales presentes en el ligante L1. A 3328 $\mathrm{cm}^{-1}$ se presenta el estiramiento $\mathrm{N}-\mathrm{H}$ de los grupos aminos; a $3046 \mathrm{~cm}^{-1}$ estiramientos $\mathrm{C}-\mathrm{H}$ del tipo sp'; en $2927 \mathrm{~cm}^{-1}$ aparecen los estiramientos $\mathrm{C}-\mathrm{H}$ alifáticos, en $1127 \mathrm{~cm}^{-1}$ aparece el estiramiento C-O. En $760 \mathrm{~cm}^{-1}$ se presentan las vibraciones de flexión correspondiente al grupo $\mathrm{NH}$ mientras que en 673 y $589 \mathrm{~cm}^{-1}$ se presentan las bandas de vibración C-H características del grupo naftaleno. La caracterización por resonancia magnética nuclear (RMN) de ${ }^{1} \mathrm{H}$ y ${ }^{13} \mathrm{C}$ confirman la estructura esperada. $\mathrm{RMN}$ de ${ }^{1} \mathrm{H}$ $\left(\mathrm{CDCl}_{3}, 400 \mathrm{MHz}\right) \delta$ en ppm: 7.0-8.2 (7H, naftalenos), 4.3 (s, $\left.2 \mathrm{H}, \mathrm{H}_{\mathrm{d}}\right), 3.68\left(\mathrm{t}, 2 \mathrm{H}, \mathrm{H}_{\mathrm{b}}\right), 3.5\left(\mathrm{~s}, 2 \mathrm{H}, \mathrm{H}_{\mathrm{a}}\right), 2.68\left(\mathrm{t}, 2 \mathrm{H}, \mathrm{H}_{\mathrm{c}}\right) . \mathrm{RMN}$ de ${ }^{13} \mathrm{C}\left(\mathrm{CDCl}_{3}, 100 \mathrm{MHz}\right) \delta$ en ppm: 120-140 (carbonos naftaleno), $70.54\left(C_{a}\right), 70.49\left(C_{b}\right), 70.27\left(C_{c}\right), 51.11\left(C_{e}\right), 48.92$ $\left(C_{d}\right)$. La figura 1 muestra la asignación de las señales para el $\mathrm{L} 1$ por RMN de ${ }^{1} \mathrm{H}$ y ${ }^{13} \mathrm{C}$. Espectrometría de masas de alta resolución (MS-ESI) arrojó la señal del ion molecular 473.2 correspondiente $[\mathrm{LH}]^{+}$y coincide para la fórmula molecular $\mathrm{C}_{30} \mathrm{H}_{36} \mathrm{~N}_{2} \mathrm{O}_{3}$. Los espectros de UV-Vis en etanol muestran dos bandas, una a $217 \mathrm{~nm}\left(\varepsilon_{\max }=2.71 \times 10^{5} \mathrm{M}^{-1} \mathrm{~cm}^{-1}\right)$ y otra banda estructurada con máximos a $271 \mathrm{~nm}\left(\varepsilon_{\text {max }}=8.7\right.$ $\left.\times 10^{4} \mathrm{M}^{-1} \mathrm{~cm}^{-1}\right), 282 \mathrm{~nm}\left(\varepsilon_{\text {max }}=1.17 \times 10^{5} \mathrm{M}^{-1} \mathrm{~cm}^{-1}\right)$ y 292 $\mathrm{nm}\left(\varepsilon_{\max }=7.85 \times 10^{4} \mathrm{M}^{-1} \mathrm{~cm}^{-1}\right)$, las cuales corresponden a transiciones $\pi-\pi^{*}$, de los anillos aromáticos en la molécula. Los espectros de excitación y emisión de fluorescencia en etanol presentan dos bandas, una centrada a $220 \mathrm{~nm}$ y otra a $276 \mathrm{~nm}$. Mientras que el espectro de emisión está constituido por dos bandas; una banda centrada a 338 $\mathrm{nm}$ correspondiente al monómero de naftaleno y otra de menor intensidad a $404 \mathrm{~nm}$, característica a la emisión de excímero de naftaleno.

Los resultados de la caracterización por las diferentes técnicas analíticas indican la obtención del producto esperado.

\section{Síntesis del ligante L1}

La síntesis de este ligante $\mathrm{M} 1$ se obtuvo mediante síntesis orgánica en fase sólida (SOFS). El ligante M1 tiene una estructura similar al ligante L1, sin embargo este ligante se encuentra soportado en una resina (resina de Merrifield), lo cual hace que el ligante sea poco soluble en la mayoría de los solventes y dificulte su caracterización por distintas técnicas. El ligante $\mathrm{M} 1$ se caracterizó a través de espectroscopia de IR y fluorescencia, además de la prueba colorimétrica de Kaiser. Primeramente, se caracterizó la resina de Merrifield por la técnica de IR; el espectro muestra las vibraciones propias del material poliestireno, el cual presenta bandas de vibración de los grupos aromáticos en las regiones de $3100-3000 \mathrm{~cm}^{-1}$ correspondientes a estiramientos $\mathrm{C}-\mathrm{H}$ del tipo $\mathrm{sp}^{2}$; en el rango de 2000-1600 $\mathrm{cm}^{-1}$ aparecen los sobretonos de los grupos aromáticos y en $1600 \mathrm{~cm}^{-1}$ los estiramientos C-C doble enlace. Por otra parte, también se observa el estiramiento $\mathrm{C}-\mathrm{Cl}$ a $874 \mathrm{~cm}^{-1}$. Al llevar a cabo la reacción de la resina de Merrifield con la etilendiamina (unidad espaciadora), el espectro de IR del producto muestra las siguientes bandas de vibración además de las bandas propias de la resina: en $3321 \mathrm{~cm}^{-1}$ aparece el estiramiento $\mathrm{N}-\mathrm{H}$ correspondiente al grupo amino de la unidad espaciadora, en $1674 \mathrm{~cm}^{-1}$ se encuentra presente la flexión del grupo $\mathrm{NH}_{2}$; además de estas bandas también se observa el estiramiento $\mathrm{C}-\mathrm{N}$ a $1180 \mathrm{~cm}^{-1}$ mientras que la banda asignada al estiramiento $\mathrm{C}-\mathrm{Cl}$ a 874 $\mathrm{cm}^{-1}$ propia de la resina desaparece. Esta reacción también se caracterizó a través de la prueba colorimétrica de káiser, la cual resultó ser positiva ya que las resinas se colorean de color azul y confirman la presencia de grupos aminos primarios en la molécula.

Posteriormente, se llevó a cabo la inmovilización de la unidad quelante, haciendo reaccionar la resina que contiene la unidad espaciadora con el reactivo bis[2-(2cloroetoxi)etil]éter. El espectro de IR del producto de la reacción presenta una banda de estiramiento $\mathrm{N}-\mathrm{H}$ a 3659 $\mathrm{cm}^{-1}$ correspondiente a los grupos aminos secundarios y la banda de estiramiento de los éteres a $1027 \mathrm{~cm}^{-1}$, lo cual indica que se llevó a cabo la reacción. Esta reacción también se monitoreó por la prueba de Kaiser con el fin de corroborar la presencia de grupos aminos primarios sin reaccionar y el resultado fue negativo, lo que indica que todos los grupos aminos primarios de la resina reaccionaron con el bis[2-(2-cloroetoxi)etil]éter. Por último se llevó a cabo la reacción de la resina modificada con el 1-naftalenmetilamina, el espectro de IR presentó las bandas de vibración antes mencionadas con la intensificación de la banda del estiramiento $\mathrm{C}-\mathrm{H}$ del tipo $\mathrm{sp}^{2}$ y de la banda $\mathrm{N}-\mathrm{H}$, además de la ausencia de la banda del estiramiento $\mathrm{C}-\mathrm{Cl}$ a $820 \mathrm{~cm}^{-1}$. De esta manera se demostró la obtención del ligante M1. Por otra parte, los espectros de excitación y emisión por fluorescencia del ligante M1 muestran que el espectro de excitación está constituido por una banda centrada a $355 \mathrm{~nm}$ y el espectro de emisión consta de una banda centrada a $386 \mathrm{~nm}$ ambas correspondientes al grupo naftaleno.

\section{Estudios de reconocimiento por fluorescencia}

\section{Estudios en solución con L1}

La evaluación de la respuesta sensora del ligante L1 con los metales $\mathrm{Li}^{+}, \mathrm{Na}^{+} \mathrm{y} \mathrm{K}^{+}$se llevó a cabo un estudio cualitativo 
de la formación del complejo. Se estudiaron adicionales a $\mathrm{Li}^{+}$los cationes $\mathrm{Na}^{+}$y K $\mathrm{K}^{+}$, porque en la recuperación del $\mathrm{Li}^{+}$ se utilizan $\mathrm{Na}_{2} \mathrm{CO}_{3}$ y $\mathrm{K}_{2} \mathrm{CO}_{3^{\prime}}$, para formar $\mathrm{Li}_{2} \mathrm{CO}_{3^{\prime}}$, por lo que es importante conocer si el ligante presenta respuesta por la presencia de estos iones [10] [11]. La figura 5 muestra los espectros de emisión del ligante L1 libre y de los complejos formados en solución. Se observó que el único ion que provocó un cambio significativo en la emisión del ligante $\mathrm{L} 1$ fue el ion $\mathrm{Li}^{+}$, el cual aumentó la emisión de $\mathrm{L} 1$ en casi un $50 \%$, en cambio la presencia de los iones $\mathrm{Na}^{+}$y K $\mathrm{K}^{+}$fue un incremento menor del $10 \%$. Por estos resultados se decidió llevar a cabo un estudio más detallado con el ion $\mathrm{Li}^{+}$.

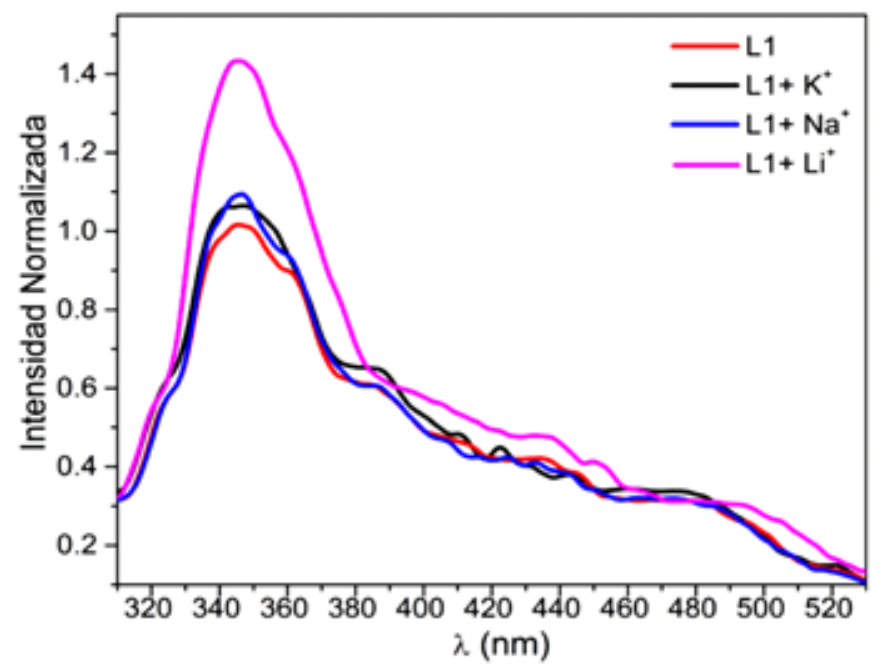

Figura 5. Prueba cualitativa del ligante $L 1$ con los metales $\mathrm{Li}^{+}, \mathrm{Na}^{+}$y K $\mathrm{K}^{+}$. L1 $\left[1 \times 10^{-6} \mathrm{M}\right]$ en buffer MOPS [0.01 $\mathrm{M}] \mathrm{a} \mathrm{pH}=7, \lambda_{\mathrm{ex}}=290 \mathrm{~nm}$.

Se llevó a cabo una titulación del ligante L1 $\left[1 \times 10^{-6}\right.$ $\mathrm{M}]$ con alícuotas de $10 \mu \mathrm{L}$ de solución de $\mathrm{LiCl}\left[3 \times 10^{-4} \mathrm{M}\right]$

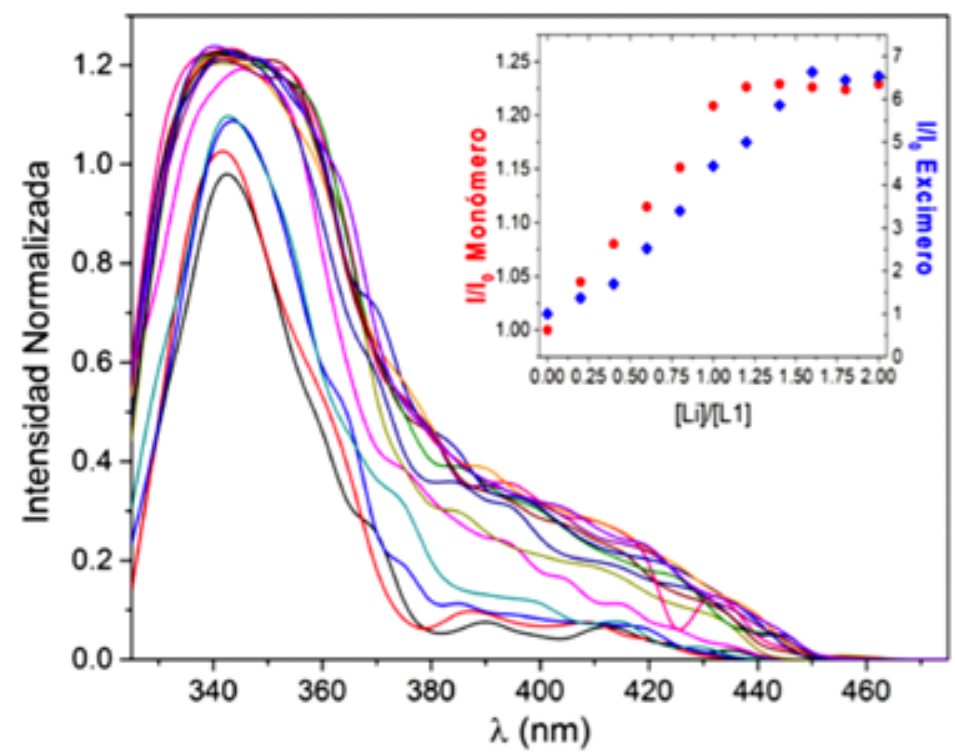

con el fin de variar la relación $\left[\mathrm{Li}^{+}\right] /[\mathrm{L} 1]$. Los espectros de este estudio se muestran en la figura 6 . Se observó que al ir adicionando la solución del metal, tanto la banda de emisión de monómero ( $335 \mathrm{~nm}$ ), como la de excímero (400 $\mathrm{nm}$ ) incrementaron su intensidad de emisión. A medida que la relación $\left[\mathrm{Li}^{+}\right] /[\mathrm{L} 1]$ cambia de 0 a 1 , la banda de emisión de monómero se hace más intensa aumentando un $20 \%$ su intensidad, lo que sugiere una estequiometria $1: 1$, (M:L). Los cambios más significativos se presentaron en la banda de emisión a $400 \mathrm{~nm}$ correspondiente a la emisión de excímero de naftaleno; esta banda aumento más de 6 veces su intensidad al formarse el complejo en solución. El aumento en la intensidad de emisión del ligante al formar el complejo se puede atribuir al aumento en la rigidez del sistema debido a la coordinación. Esto provoca un aumento en la eficiencia cuántica del sistema ya que disminuyen las rutas vibracionales para el decaimiento no radiativo. Otro factor que opera en el sistema estudiado, y que contribuye al incremento en la intensidad de emisión de excímero, es el cambio conformacional. Al formarse el complejo, el ligante L1 cambia su conformación, permitiendo un arreglo en que los anillos de naftaleno se acercan originando una interacción del tipo $\pi-\pi$, formación del excimero de naftaleno. En la figura 6 se representa un posible arreglo de la formación del complejo. De los cambios observados en los espectros de emisión por la presencia de litio, se determinó el límite de detección en el sistema, tanto en la banda de monómero como la de excímero, encontrando valores de $1.6 \times 10^{-8} \mathrm{M}$ y $3.9 \times 10^{-8}$ $M$ respectivamente. En ambas bandas el sistema presenta linealidad hasta concentración de $1 \times 10^{-6} \mathrm{M}$. Lo destacado del sistema sintetizado en este trabajo es que la molécula sensora es un ligante abierto del tipo biscromofórico ya que la mayoría de los sensores para litio son del tipo macrocíclico, algunos de ellos con muy buenas respuestas [12].

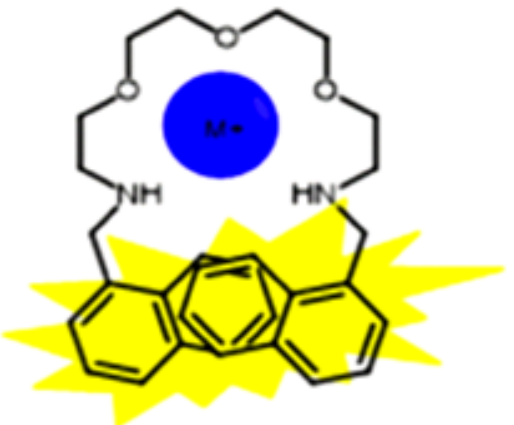

Figura 6. Espectro de emisión del ligante L1 a diferentes concentraciones de $\mathrm{Li}^{+}$. $\mathrm{L} 1\left[1 \times 10^{-6} \mathrm{M}\right]$ en buffer MOPS [0.01 M] a pH $=7, \lambda_{\text {ex }}=290 \mathrm{~nm}$. Inserto: Efecto de la concentración del metal sobre la intensidad de fluorescencia del excímero a $\lambda_{\mathrm{ex}}=400 \mathrm{~nm}$. 


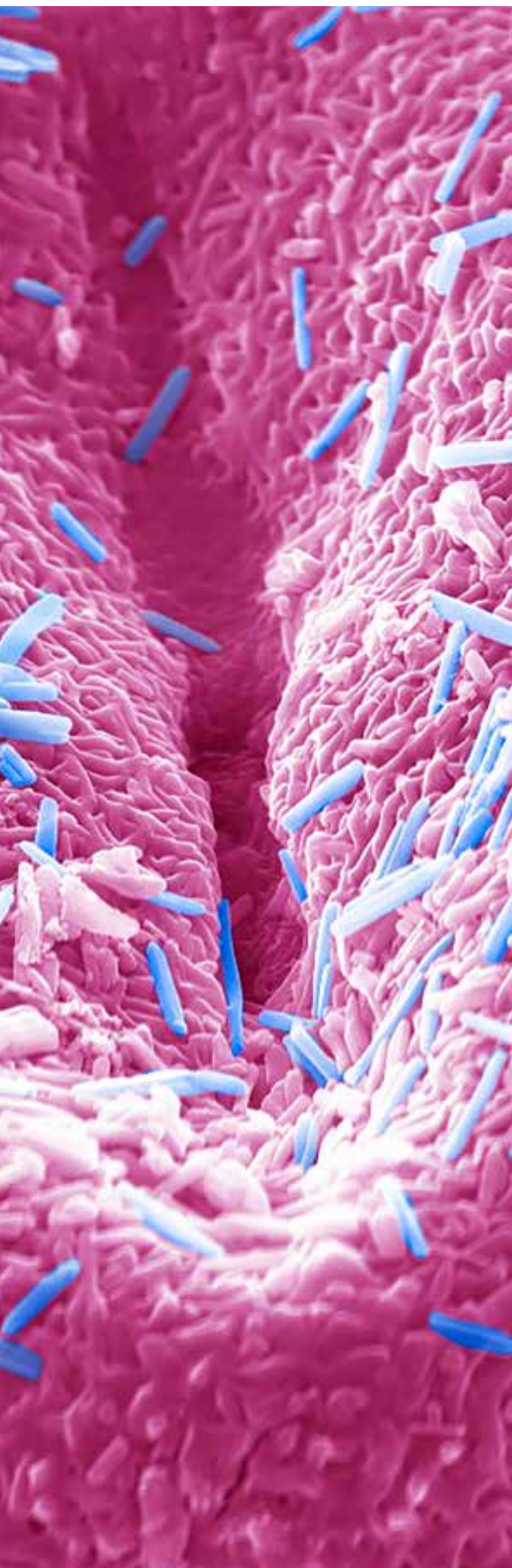

\section{Estudios en fase sólida con M1}

Se realizó un estudio cualitativo de la respuesta sensora del ligante $M 1$ con los metales $\mathrm{Li}^{+}, \mathrm{Na}^{+}$y K${ }^{+}$, el cual consistió en colocar de 12-13 mg de M1 en una celda de flujo, posteriormente se le hizo pasar agua deionizada y se tomó el espectro. A continuación se le hizo pasar la solución del catión a evaluar ([0.01 M], $\mathrm{pH}=7$ ) por varios minutos para asegurar que todos los sitios del material M1 tuvieran contacto con el metal y posterior a esto se adquiere el espectro. Posteriormente, se hizo pasar por varios minutos agua deionizada y nuevamente se adquirió el espectro. Se encontró respuesta para el catión $\mathrm{Li}^{+}$y $\mathrm{Na}^{+}$y una respuesta nula para $\mathrm{K}^{+}$.

Con la finalidad de determinar la respuesta sensora hacia los cationes $\mathrm{Li}^{+}$y $\mathrm{Na}^{+}$se realizó un estudio que consistió en inyectar $100 \mu \mathrm{L}$ de la solución del metal a evaluar, con una concentración de $1.5 \times 10^{-3} \mathrm{M}$, a un sistema de flujo; cuando la solución del metal llegó a la zona de lectura, se adquirió el espectro de emisión. Este procedimiento se repitió hasta que se observó que la emisión se mantuvo constante. Las figuras 7 y 8 muestran los espectros de emisión del ligante M1 con las diferentes inyecciones de las soluciones de $\mathrm{Li}^{+}$y Na${ }^{+}$, respectivamente, utilizando una $\lambda_{\text {ex }}$ de 355 $\mathrm{nm}$. La figura 7 correspondiente a las inyecciones de $\mathrm{Li}^{+}$, muestra que a partir de la concentración de $1 \times 10^{-6} \mathrm{M}$, la emisión del M1 se incrementó en forma gradual hasta una concentración de $3.6 \times 10^{-6} \mathrm{M}$; posterior a esta concentración el sistema se mantuvo constante. En el recuadro de la figura 7 se indica como cambió la intensidad de fluorescencia a $400 \mathrm{~nm}$ a diferentes concentraciones de $\mathrm{Li}^{+}$. Algo importante encontrado en este sistema de estudio es que el metal se une fuertemente a la unidad quelante, es decir que M1 además de indicar la presencia del metal también se puede utilizar para remover el metal en una muestra, cuando se presenta este tipo de respuesta se dice que el material presenta respuesta de dosímetro. Se determinó el límite de detección de este sistema y se encontró $1.67 \times 10^{-7} \mathrm{M}$.

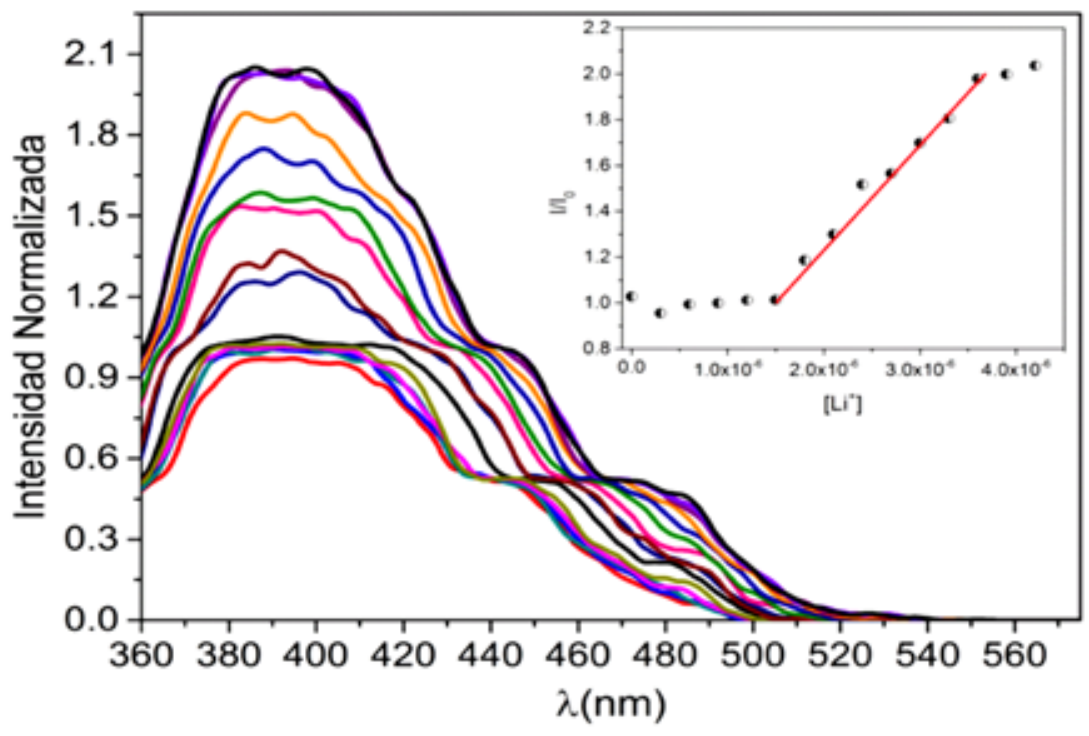

Figura 7. Espectros de emisión del ligante $\mathrm{M} 1$ a diferentes inyecciones de solución de $\mathrm{Li}^{+}$, se utilizó una $\lambda_{\text {ex }}$ de $355 \mathrm{~nm}$. Inserto: Variación de la intensidad de emisión a $400 \mathrm{~nm}$.

La figura 8 muestra los cambios obtenidos en la emisión del ligante M1 en presencia del ion $\mathrm{Na}^{+}$. Los cambios observados no muestran un comportamiento lineal a medida que se fue aumentando la concentración 
de $\mathrm{NaCl}$, sin embargo la emisión aumentó hasta alcanzar el doble de la emisión inicial. En este sistema también se encontró una respuesta de dosímetro.

Al comparar los resultados de los estudios de coordinación de L1 y M1 con litio, se encontró que los límites de detección son menores en L1 y esto es debido a que se utiliza menor concentración del ligante, los resultados obtenidos indican que este sistema se puede utilizar para cuantificar la cantidad de litio presente en una muestra; sin embargo, la muestra M1 puede ser utilizada para la remoción de litio en aguas contaminadas con dicho metal.

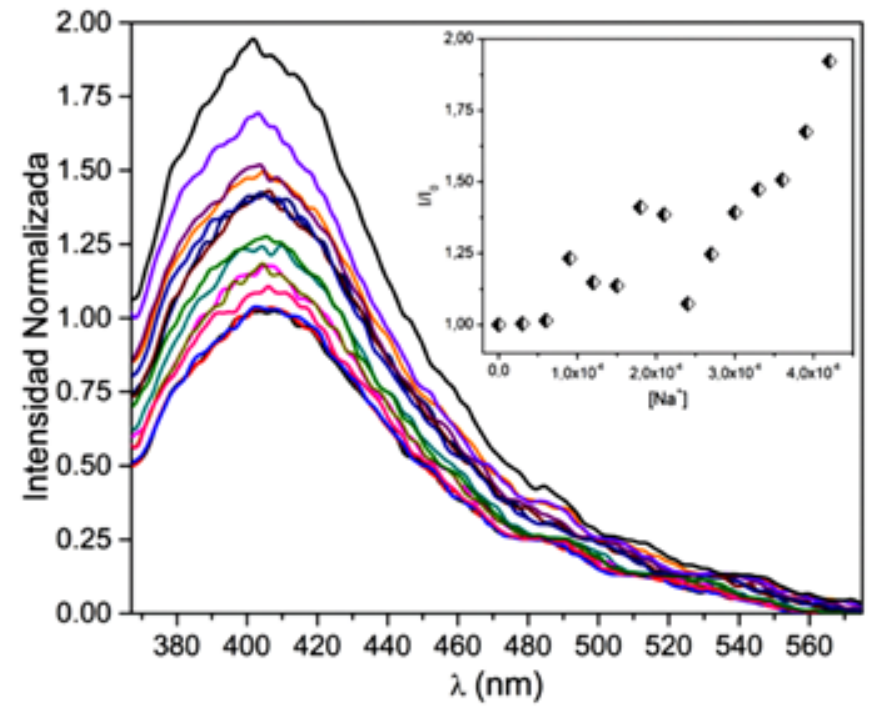

Figura 8. Espectros de emisión del ligante $\mathrm{M} 1$ a diferentes inyecciones de solución de $\mathrm{Na}^{+}$, se utilizó una $\lambda_{\text {ex }}$ de $355 \mathrm{~nm}$. Inserto: Variación de la intensidad de emisión a $400 \mathrm{~nm}$.

\section{CONCLUSIONES}

Se sintetizó un nuevo ligante fluorescente biscromofórico (L1) a partir de la reacción entre el bis[2-(2cloroetoxi)etil]éter con la amina 1-naftalen-il-metilamina, se obtuvo con un rendimiento del $85 \%$. La caracterización del ligante $\mathrm{L} 1$ mediante IR, RMN de ${ }^{1} \mathrm{H}$ y ${ }^{13} \mathrm{C}$, además de espectrometría de masas, confirman la formación y pureza del producto.

La evaluación de la respuesta sensora del ligante L1 por espectroscopía de fluorescencia en solución MOPS a $\mathrm{pH} 7$, provocó un aumento en la emisión tanto de excímero como de monómero de naftaleno. La intensificación de la banda de excímero indica que al ocurrir la formación del complejo, los anillos de naftaleno adquieren un arreglo cara a cara. Este ligante se puede utilizar para cuantificar $\mathrm{Li}^{+}$en aguas contaminadas.

Se obtuvo el ligante M1 por la técnica de SOFS utilizando como soporte la resina de Merrifield y se evaluó su capacidad como sensor fluorescente para los iones $\mathrm{Li}^{+}$, y se encontró que el material presenta respuesta de dosímetro para $\mathrm{Li}^{+}$, lo cual indica que se puede utilizar para removerlo en una muestra acuosa.

\section{AGRADECIMIENTOS}

Este trabajo fue financiado por el Consejo Nacional de Ciencia y Tecnología de México (CONACYT), Proyecto 214524, Resinas Funcionalizadas para retener iones metálicos de aguas contaminadas.

\section{BIBLIOGRAFÍA}

[1] J. W. Steed, D. R. Turner, and K. Wallace, Core concepts in supramolecular chemistry and nanochemistry: John Wiley \& sons, 2007.

[2] J. W. Steed, and J. L. Atwood, Supramolecular chemistry: John Wiley \& sons, 2013.

[3] E. V. Basiuk, J. G. Lara, and V. A. Basiuk, “Química supramolecular de los compuestos obtenidos por la combinación entre los macrociclos poliaza y los ácidos aromáticos," Journal of the Mexican Chemical Society, vol. 44, no. 4, pp. 289-293, 2000.

[4] A. Antelo Queijo, Arquitecturas supramoleculares generadas por nuevos derivados de ciclodextrinas y ácidos biliares: Univ. Santiago de Compostela, 2008.

[5] J. S. Martínez, "Síntesis y evaluación de la capacidad complejante de ligandos nitrogenados derivados de bifenilos sustituidos," 2007.

[6] O. M. D. L. Salud, "Guía breve de métodos analíticos para determinar las concentraciones del plomo en la sangre," 2013.

[7] M. Velázquez, and D. Yolotzin, "Recuperación de litio a partir de pilas de desecho por métodos hidrometalúrgicos," 2012.

[8] J. Ströbele-Gregor, "El proyecto estatal del litio en Bolivia," Nueva Sociedad, vol. 244, 2013.

[9] J. Calé, and R. J. Lutfi, "El litio y su influencia en la función tiroidea," Revista Argentina de Endocrinología y Metabolismo, vol. 46, no. 3, pp. 37-42, 2009.

[10]l. Dai, I. Wigman, and K. Zhang, "Sensitive and direct determination of lithium by mixed-mode chromatography and charged aerosol detection," Journal of Chromatography a, vol. 1408, pp. 87-92, 2015.

[11]S. Misra, and P. N. Froelich, "Measurement of lithium isotope ratios by quadrupole-icp-ms: application to seawater and natural carbonates," Journal of Analytical Atomic Spectrometry, vol. 24, no. 11, pp. 1524-1533, 2009.

[12]G. R. C. Hamilton, S. K. Sahoo, S. Kamila, N. Singh, N. Kaur, B. W. Hyland, and J. F. Callan, "Optical probes for the detection of protons, and alkali and alkaline earth metal cations," Chemical Society Reviews, vol. 44, no. 13, pp. 4415-4432, 2015.

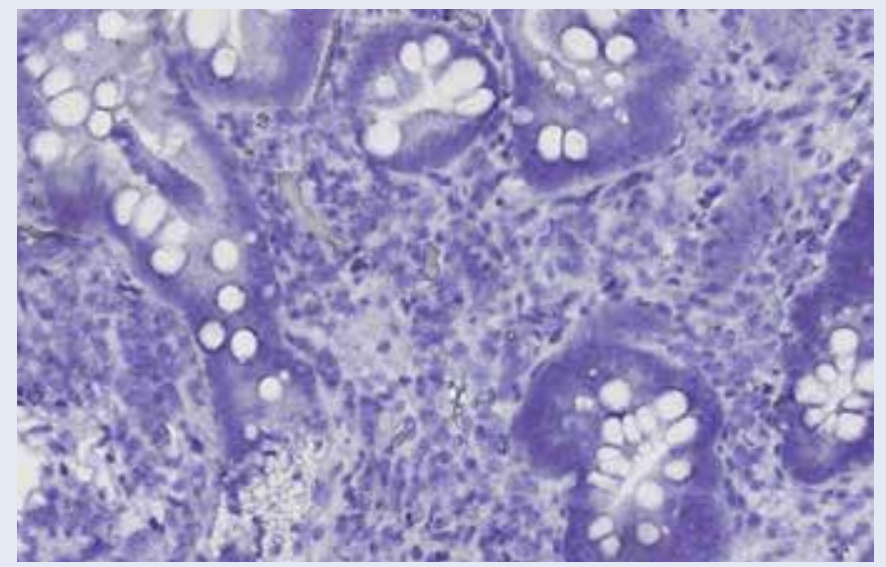

